\title{
Capacitive fundamental power coupler and pickup for the 56 MHz SRF cavity
}

\author{
E. M. Choi and H. Hahn
}

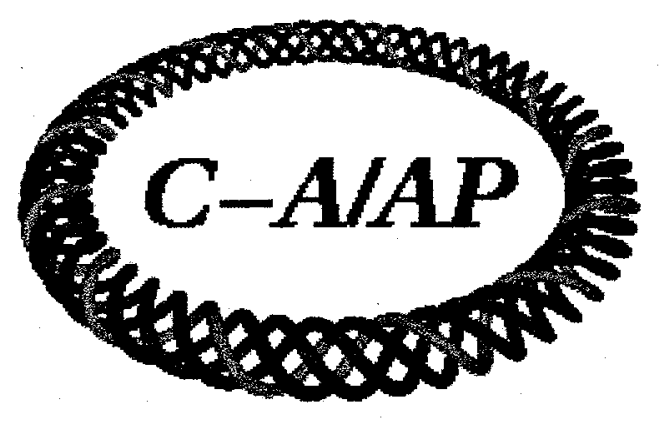

\section{Collider-Accelerator Department Brookhaven National Laboratory \\ Upton, NY 11973}

Notice: This document has been authorized by employees of Brookhaven Science Associates, LLC under Contract No. DE-AC02-98CH10886 with the U.S. Department of Energy. The United States Government retains a non-exclusive, paid-up, irrevocable, world-wide license to publish or reproduce the published form of this document, or allow others to do so, for United States Government purposes. 


\title{
Capacitive fundamental power coupler and pickup for the $56 \mathrm{MHz}$ SRF cavity
}

\author{
E. M. Choi and H. Hahn
}

July 16, 2008

\begin{abstract}
The beam excited $56 \mathrm{MHz}$ SRF cavity will have a power coupler for a fast frequency tuner. The calculation shows the coupling of the power coupler, $\beta_{o p t}$, is around 50 . Size and location of the power coupler are determined by measurements. Measurements are in good agreement with the simulation results. The axial location of the power coupler for the $\mathrm{Nb}$ cavity is limited by corrugations made on the cavity outer conductor for purpose of removing any multipacting. The preferred axial location is $14.5 \mathrm{~cm}$ away from the cavity gap start where a slow tuner plate will be. MWS simulations are done to determine the length of the power coupler inner conductor and pickup probe for the $\mathrm{Nb}$ cavity at the fixed axial location. Size and location of both the fundamental power coupler and the pickup probe can be decided from the simulation results.
\end{abstract}

\section{Introduction}

The $56 \mathrm{MHz}$ Superconducting cavity is going to be installed in RHIC for enhancing the luminosity. Table 1 shows the cavity parameters of the SRF $56 \mathrm{MHz}$ cavity. A $1 \mathrm{~kW}$ of power amplifier is chosen for a fast frequency tuning, which is capable of providing $\pm 0.75 \mathrm{~Hz}$ frequency tuning range for compensating microphonics. The power coupler will be capacitively coupled near the cavity gap region. The required coupling factor for the fundamental power coupler (FPC) was $\beta_{\text {opt }}$ of 50 calculated by Ilan Ben-Zvi, which in turn corresponds to $\mathrm{Q}_{\text {ext }}$ of $4 \times 10^{7}$ assuming $\mathrm{Q}_{0}$ is $2 \times 10^{9}$.

As for a pickup probe (PU), $1 \mathrm{~W}$ power coming out from the pickup probe will be enough for output signal which corresponds to external $Q$ of $8 \times 10^{10}$. Size and locatioin of both the FPC and the PU are determined by measurements and also the results are compared to simulation results for projecting it onto the $\mathrm{Nb}$ cavity case.

Table 1: Summary of the $56 \mathrm{MHz}$ SRF cavity parameters

\begin{tabular}{cc}
\hline \hline Parameter & Values \\
\hline Frequency & $56.25 \mathrm{MHz}$ \\
Voltage & $2.5 \mathrm{MV}$ \\
Superconducting surface resistance & $10 \mathrm{n} \Omega$ \\
Power dissipated to LHe $(4.2 \mathrm{~K})$ & $42 \mathrm{~W}$ \\
Q factor & $1.8 \times 10^{9}$ \\
Stored energy & $221 \mathrm{~J}$ (From MWS) \\
R over Q (accelerator definition) & 79.9 (From MWS) \\
\hline \hline
\end{tabular}




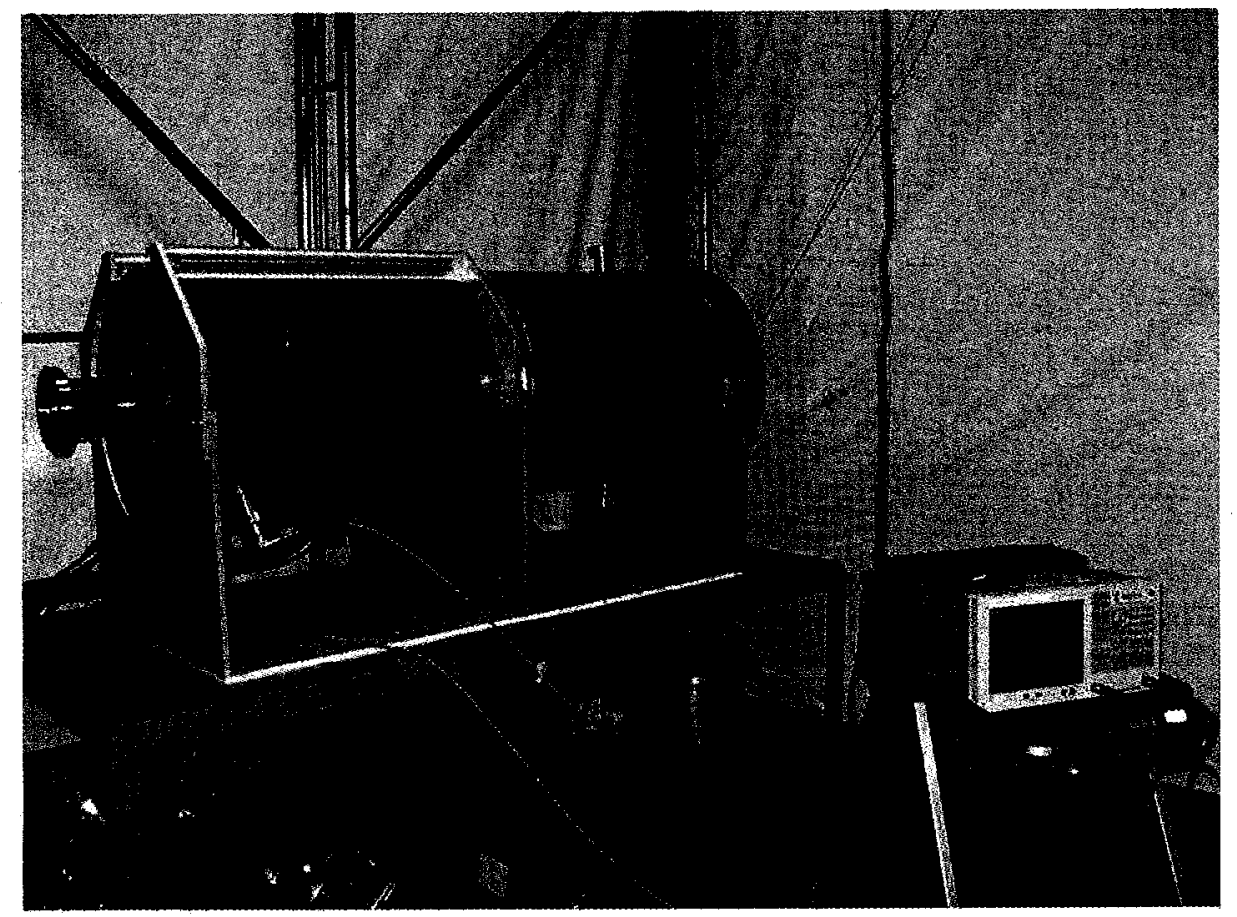

Figure 1: The experimental setup of the Cu prototype cavity

\section{Experimental setup}

Fig. 1 shows the measurement setup for the Cu prototype cavity with a Vector Network Analyzer (Agilent E5071C, $9 \mathrm{kHz}-8.5 \mathrm{GHz}$, ENA Series Network Analyzer). Measurements on the $\mathrm{Cu}$ prototype for the FPC and the PU were done in the pickup probe ports of the prototype cavity, which are $20 \mathrm{~cm}$ away from the cavity gap start. The schematic of the prototype Cu cavity is shown in Fig. 2. The diameter of the FPC probe is $1.52 \mathrm{~cm}$ for the test and the length of the FPC probe is varied so that the position for giving the required external $\mathrm{Q}$ can be searched.

\section{The interpretation of external Q measurements via scattering parameters}

The measurements were done in two different ways. One uses $\mathrm{S}_{11}$ measurement and the other uses $S_{21}$ with an network analyzer. If the coupling between the cavity and the load is not too small to pick up some signal, $\mathrm{S}_{11}$ measurements can be used to obtain external $\mathrm{Q}$ factor accurately enough. Fig. 3 shows the circuit representation of the cavity with external loads using the ideal transformer. Here, the external loads are vector network analyzer input and output ports. The circuit components are normalized. $X$ equals to 


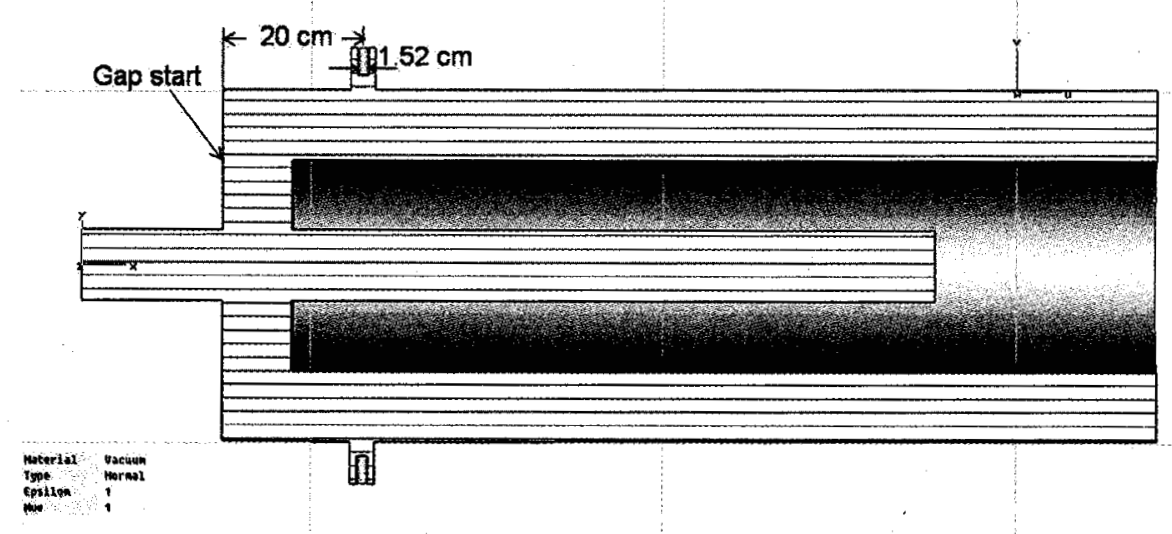

Figure 2: A drawing of the prototype cavity with PU probes. The gap start is specified in the drawing where the slow tuner will be.

$\left(\omega / \omega_{0}-\omega_{0} / \omega\right)$. Fig. 4 is the equivalent circuit of Fig. 3 referred to the middle loop. The $\mathrm{S}_{21}$ is defined as

$$
S_{21}=\frac{2 V_{2}}{V_{0}}
$$

The current $i$ in Fig. 4 can be expressed as follows.

$$
\sqrt{\beta_{i n}} V_{0}=i \cdot\left[\frac{\beta_{\text {in }}}{Q_{0}}+\frac{1}{Q_{0}}+j X+\frac{\beta_{\text {out }}}{Q_{0}}\right]=i \cdot\left[\frac{1}{Q_{L}}+j X\right]
$$

Here, $\frac{1}{Q_{L}}=\frac{1}{Q_{0}}+\frac{\beta_{i n}}{Q_{0}}+\frac{\beta_{\text {out }}}{Q_{0}} . V_{2}$ expression can be related to the current $i$ showing in Eq. 3 .

$$
\sqrt{\beta_{o u t}} V_{2}=\frac{\beta_{o u t}}{Q_{0}} \cdot i
$$

Using Eq. 2 and Eq. 3, $\mathrm{S}_{21}$ defined in Eq. 1 can now become as follows.

$$
S_{21}=\frac{2 \sqrt{\beta_{i n}} \sqrt{\beta_{o u t}}}{Q_{0}\left(\frac{1}{Q_{L}}+j X\right)}
$$

Knowing that $\beta_{\text {in }}=Q_{0} / Q_{\text {in }}$ and $\beta_{\text {out }}=Q_{0} / Q_{\text {out }}$, one can construct $\mathrm{S}_{21}$ as follows.

$$
S_{21}=\frac{2 Q_{L}}{\sqrt{Q_{\text {in }} Q_{\text {out }}}\left(1+j X Q_{L}\right)}
$$

At critically coupled case, $\mathrm{Q}_{e x t}$ equals to $\mathrm{Q}_{0}$. Therefore, $\mathrm{Q}_{L}$ is half of $\mathrm{Q}_{0}$. One can find external $Q$ factor by measuring $S_{21}$ using the following equation from Eq. 5 at critically coupled case $\left(\mathrm{Q}_{L}=\mathrm{Q}_{0} / 2, \mathrm{Q}_{i n}=\mathrm{Q}_{0}\right.$, and $\left.\mathrm{Q}_{\text {out }}=\mathrm{Q}_{e x t}\right)$ at resonance $(X=0)$.

$$
Q_{e x t}=\frac{Q_{0}}{S_{21}^{2}}
$$




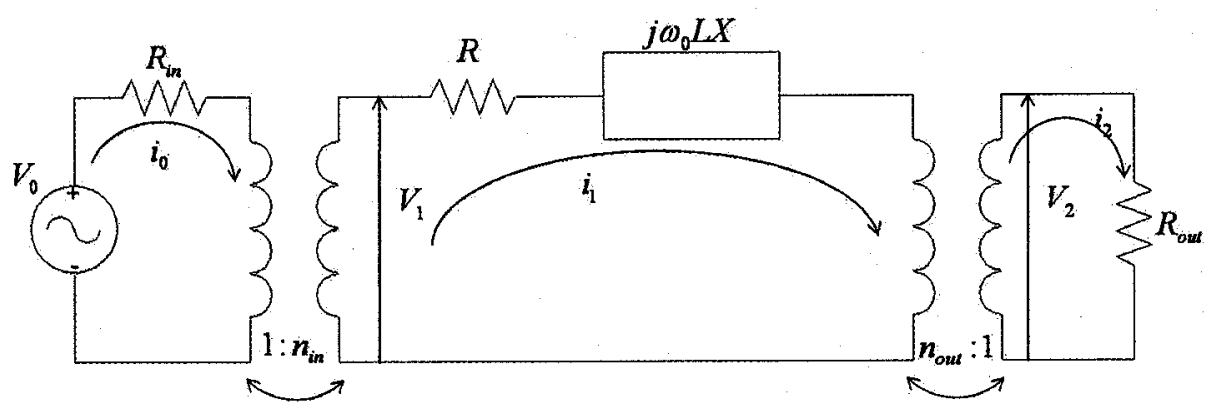

Figure 3: The equivalent circuit representaion of the cavity with an ideal transformer

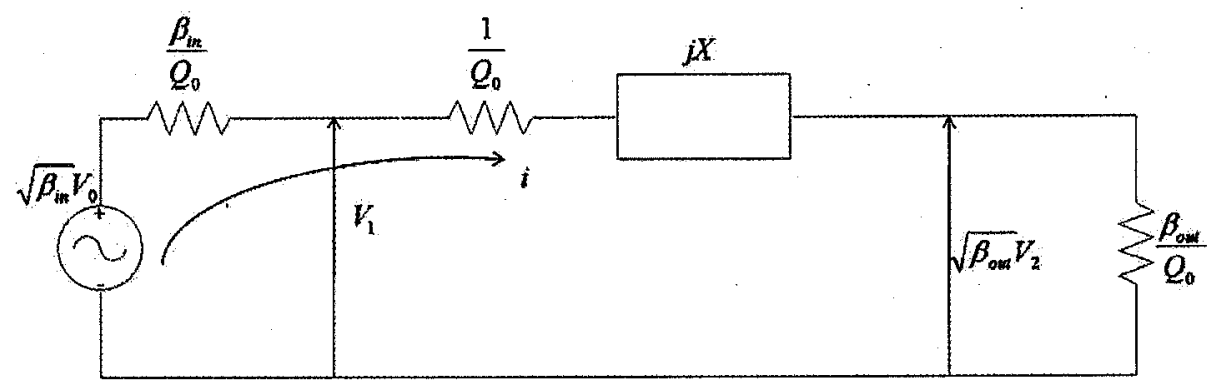

Figure 4: The equivalent circuit representaion of the cavity referred to the middle cavity loop

Eq. 6 is a direct expression for obtaining $Q_{e x t}$ from the $S_{21}$ measurements.

$\mathrm{S}_{11}$ can be expressed as follows.

$$
S_{11}=\frac{R+j \omega_{0} L X-Z_{0}}{R+j \omega_{0} L X+Z_{0}}=\frac{1-\beta_{\text {in }}+\beta_{\text {out }}+j X Q_{0}}{1+\beta_{\text {in }}+\beta_{\text {out }}+j X Q_{0}}
$$

One can get $\beta_{\text {out }}$ from the $S_{11}$ measurement at resonance. In the measurement, we have $\beta_{i n}=0$ by having the pickup port open. Therefore, the coupling of the power coupler to the cavity is now easily obtained from $\mathrm{S}_{11}$.

$$
\beta_{\text {out }}=\frac{Q_{0}}{Q_{e x t}}
$$

Eq. 8 gives $Q_{e x t}$ value. However, if the coupling gets too weak, the $S_{11}$ does not give an accurate measurement. A more accurate measurement is directly using $S_{21}$ parameter at critically coupled $\beta_{i n}$.

\section{The Fundamental power coupler study}

The critically coupled case was achieved by having a proper size of the probe and penetration to the cavity. The probe whose diameter is $0.64 \mathrm{~cm}$ is $3.8 \mathrm{~cm}$ penetracted into the cavity and it has a $3.18 \mathrm{~cm}$ diamter disk on the top of the probe. The measured $Q_{0}$ 


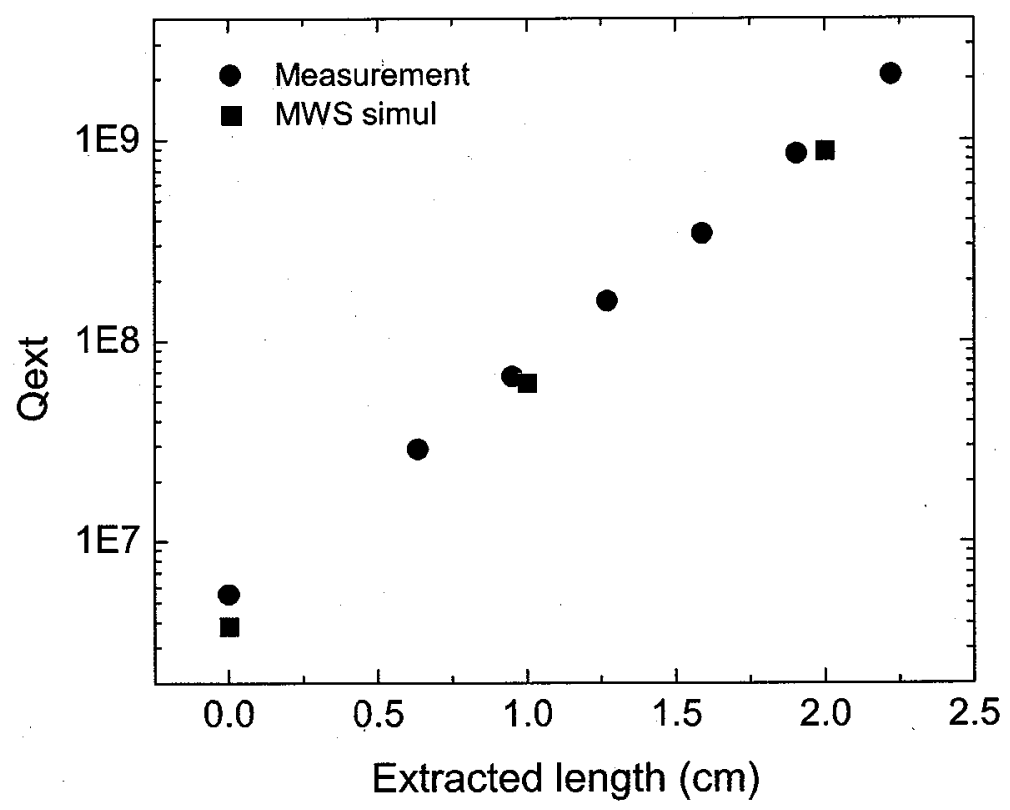

Figure 5: Experimental external Q values compared to the MWS simulation results. Black circle represents eperimental data and red rectangula represents simulation data.

is approximately 8400 for the $\mathrm{Cu}$ prototype. For the test of the FPC, the length of the FPC probe was varied while the diameter is fixed to be $1.52 \mathrm{~cm}$ as shown in Fig. 2.

Fig. 5 shows the result of external Q measurement compared to the MWS simulation results modeled as shown in Fig. 2. The length of the FPC inner conductor was varied and the tip of the FPC inner conductor was extracted to the outside of the cavity for each point because even at no penetration $Q_{e x t}$ value is much smaller than the required $Q_{e x t}$. As shown in the Fig. 5, the experimental data matches very well to the simulation data by MWS. The $Q_{e x t}$ of $4 \times 10^{7}$ is achieved around $1 \mathrm{~cm}$ extracted length.

The measurement was made at $20 \mathrm{~cm}$ away from the gap start of the prototype $\mathrm{Cu}$ cavity. The planned location for the FPC is at either the center of the cavity gap or $14.5 \mathrm{~cm}$ away from the gap start in the $\mathrm{Nb} \mathrm{SRF}$ cavity. In order to circumvent possible cryogenic design difficulties, $14.5 \mathrm{~cm}$ away from the gap start is a better location for the FPC as shown in Fig. 6. The $14.5 \mathrm{~cm}$ location is determined such that the FPC can be situated between the first corrugation and the next corrugation (corrugation of the outer conductor is applied in order to prevent multipacting.). Since the prototype cavity does not have an open coupler port for various axial locations, the MWS simulation study determines the port size and the length of the FPC (Notice that the simulation data agrees well to the experment as shown in Fig. 5.). The size of the port for the new FPC location is limited by the corrugations of the cavity surface and the maximum opening 


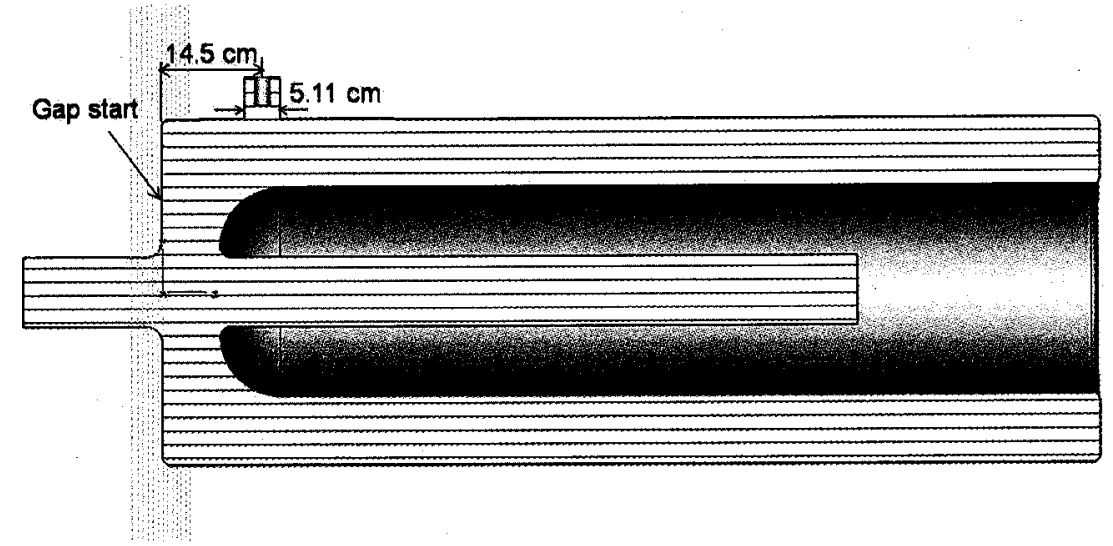

Figure 6: A drawing of the $\mathrm{Nb}$ cavity with a FPC.

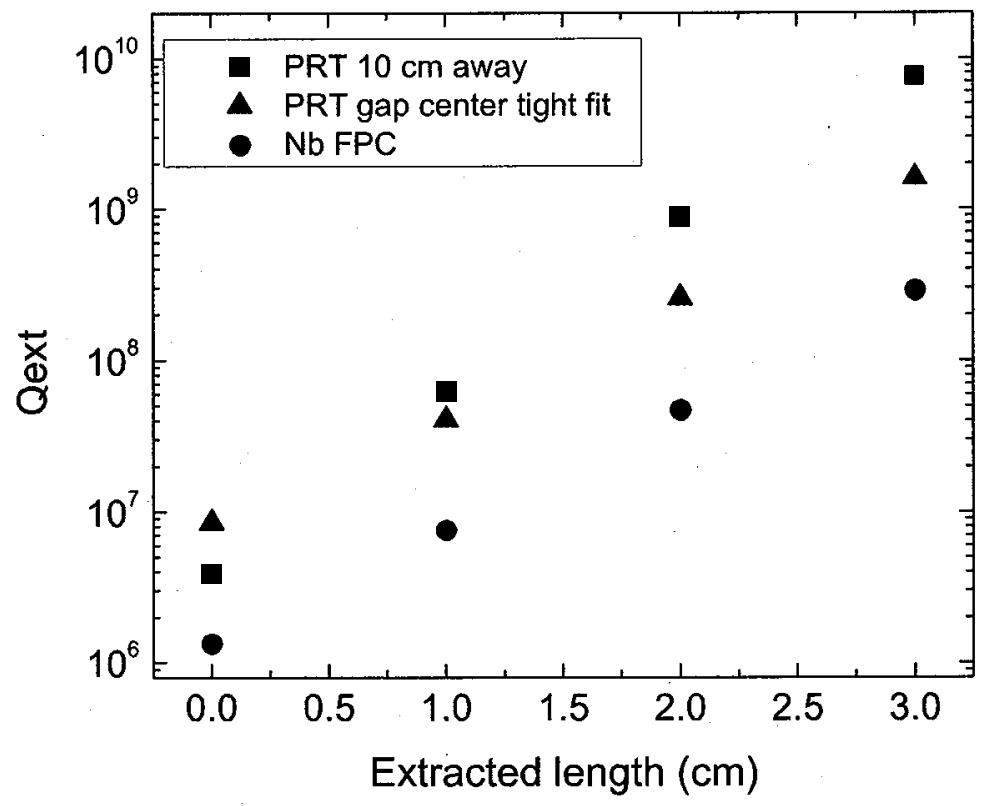

Figure 7: Simulation study on the external Q values. Black circle, blue triangle, and red circle represent MWS simulation of current setup, simulation of a new location at the gap center with a maximum port size and $\mathrm{Nb}$ cavity with a new location and a maximum port size, respectively. 
for the FPC port is $5.11 \mathrm{~cm}$. The FPC probe size is determined to be $2.21 \mathrm{~cm}$ obtained from the $50 \Omega$ relationship.

Fig. 7 shows the MWS simulation study on the external Q values as the FPC size and length are varied. When the FPC is at the gap center of the prototype cavity with the diameter of the probe of $2.21 \mathrm{~cm}, 1 \mathrm{~cm}$ extration will give the desired $Q_{\text {ext }}$. Projection onto the $\mathrm{Nb} \mathrm{SRF}$ cavity is made in Fig. 7 with red circles. The FPC is located $14.5 \mathrm{~cm}$ away from the gap start, and the FPC probe diameter is $2.21 \mathrm{~cm} .2 \mathrm{~cm}$ extraction will result in the target external $\mathrm{Q}$ of $4 \times 10^{7}$. It should be pointed out that the capacitively coupled FPC probe has a feature that allows the extraction length to be variable so that it is capable of changing the coupling between the cavity and the FPC in case of allowing higher FPC power to handle higher microphonics frequency than $\pm 0.75 \mathrm{~Hz}$.

\section{The Pickup Probe study}

The size and length of the PU are determined based on the MWS simulation result. The PU is located at the same vertical plane as the FPC, which is $14.5 \mathrm{~cm}$ away from the gap start. The inner diameter of the PU port is chosen to be $1.9 \mathrm{~cm}$, and the diameter of the PU probe is determined by $50 \Omega$ relationship with the PU port, which is $0.83 \mathrm{~cm}$. Fig. 8 shows the external $Q$ values as a function of the extracted length of the PU. From the simulation, the PU probe is to be extrated by around $2.5 \mathrm{~cm}$ from the cavity surface.

\section{Conclusion}

$1 \mathrm{~kW}$ power coupler will act as a fast tuner. The location and the size of the fundamental power coupler are decided based on the measurements and simulations. The external Q of the power coupler from the calculation is $4 \times 10^{7}$ from the coupling $\beta$ of 50 . Measurements from the $\mathrm{Cu}$ prototype were made using the currently available pickup ports. The results were compared to the MWS simulation and give an excellent agreement between experiments and simulation. For the Nb SRF cavity, corrugations on the cavity outer cylinder, which are made to prevent possible multipacting, limit the possible FPC location. It is considered to be favorable to have the FPC $14.5 \mathrm{~cm}$ away from the gap start (Around the gap center, there will be a slow tuner and helium vessel, which will make somewhat complicated to have the power coupler in there.). From the MWS simulation, one can determine the length of the FPC probe in the Nb SRF cavity. The PU probe size and location can be also determined from the MWS result.

\section{Acknowledgement}

The authors would like to thank Ilan Ben-Zvi for calculation of the coupling factor for the FPC and reading the manuscript. 


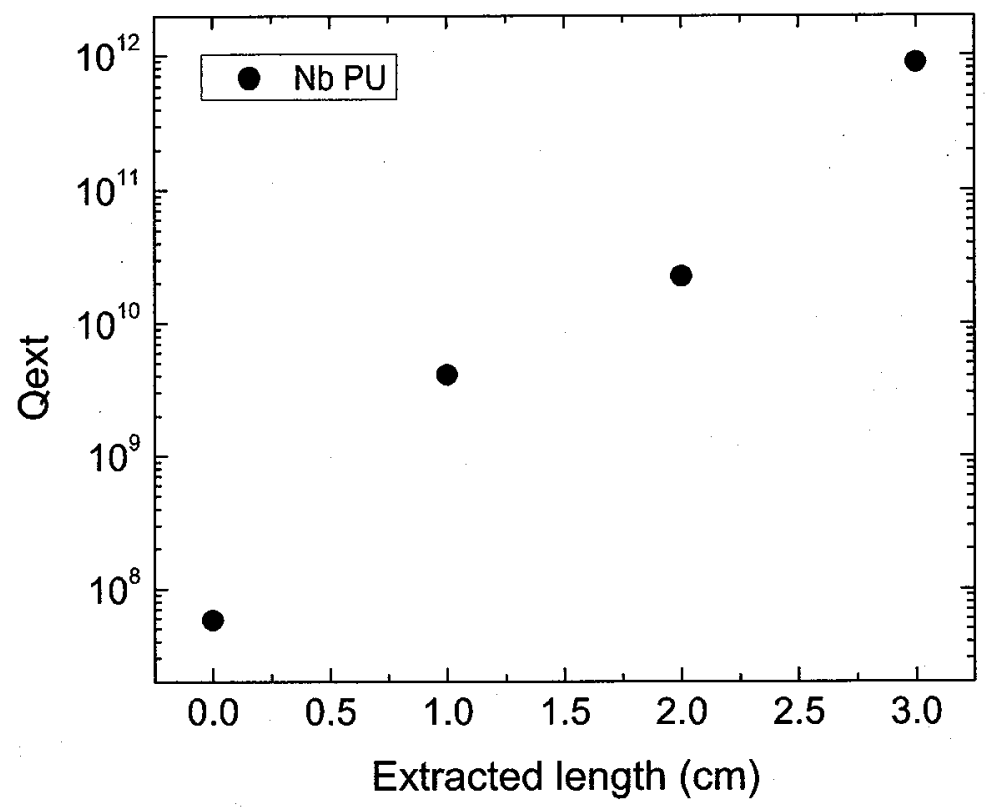

Figure 8: Simulation study on the PU probe. 